\title{
Mesh Networks
}

Paolo Cardullo, National University of Ireland Maynooth and Denis Jaromil Roio, Dyne.org Foundation

paolo.cardullo@mu.ie

\section{Word count: 1194}

Abstract: Wireless Mesh Networks have the potentials to establish and maintain ad-hoc connectivity and share gateway access to other networks with an array of mobile end-users (and their various requests, services and applications), while typically featuring a minimal and inexpensive infrastructure. While more recent applications of WMNs concern the home space or the urban milieu, where smart devices are increasingly common, WMNs became popular for extending Internet connectivity over Wi-Fi to low-income or rural communities. The following entry focuses on these two applications, highlighting advantages, actors and issues.

Keywords: Wireless Mesh Networks, Wi-Fi, Community Networks, IoT

Wireless Mesh Networks (WMNs) are decentralised network systems where communication paths across nodes can change dynamically and asynchronously thanks to a routing algorithm and according to different conditions, e.g. latency or proximity, which make the network particularly resilient to outages. Thus, WMNs have the potentials to establish and maintain ad-hoc connectivity and share gateway access to other networks with an array of mobile endusers (and their various requests, services and applications), while typically featuring a minimal and inexpensive infrastructure.

The most popular operating system used for setting up WMNs is OpenWRT, a minimal port of the Linux kernel on ARM boards using the Busybox system tools. Historically, the hardware devices receiving most support are the Linksys WRT routers (in particular the WRT54G series) because they could be easily repurposed with a different operating system than the one provided by the vendor. For the mesh routing the most popular software implementation is OLSRD (Optimized Link State Routing Protocol Daemon), initially compliant with RFC3626, and B.A.T.M.A.N. which is still actively developed. 
The mesh remains a network whether or not it is connected to the Internet. However, if gateway routers are present, any node routed by the same WMN can access the Internet. While more recent applications of WMNs concern the home space or the urban milieu, where smart devices are increasingly common, WMNs became popular for extending Internet connectivity over Wi-Fi to low-income or rural communities. The following sections focus on these two applications, highlighting advantages, actors and issues.

\section{Community Wireless Mesh Networks}

CWMNs have been under development since early 2000s thanks to the work of ethical hackers and activists responding to a series of local needs and generally advocating a more open, neutral and democratic Internet [1]. Thanks to their inexpensive hardware and relative ease of assemblage, CWMNs have been deployed in many ways, operating as specialised solutions to local issues: therefore, each case is specific and related to its wider digital and political ecosystem.

Generally, CWMNs have been an answer to excessive connectivity costs and slow service, since they allow communities to share the cost of network deployment, use of resources and provision of services, such as community radios, data sharing applications or listing of community events. For instance, both Ninux in Italy and Freifunk in Germany started in 2002 and grew to operate about 40k nodes; while SNet in Havana has expanded to 20k users who play networked games and exchange electronic items, although not offering a gateway to the public Internet since connectivity in Cuba is patchy and limited to public parks and schools (because of the US embargo and restrictive local policies) [2]. Conversely, adoption of CWMNs seems to have regained traction in the US because of the current Net Neutrality debate, where Big Cable (quasi-monopolistic Internet Service Providers) have been lobbying for selective throttling, slowing down and metering, especially in the fibre-to-home market. Obviously, CWMNs have been providing last-mile access to remote communities where the market 'failed': this is how Guifi.net started in Catalonia in 2004, now operating up to 33k nodes.

CWMNs are deployed also to fight data extractive practices since mesh are, ultimately, Intranet systems with locally controlled repositories of data. Crowd-shared WMNs in dense 
residential areas, in fact, can enable resource pooling and commoning across the Internet (filesharing, game modding, data repositories). The resilience of WMNs has been occasionally tested by natural or man-made disasters. When Hurricane Sandy destroyed most of the communication infrastructure in NYC, Red Hook mesh functioned as an effective back-up in the area. However, since WMNs operate in unlicensed frequency bands which are subject to uncontrolled interference from a range of sources and overcrowding, their efficacy during emergency is disputed [3].

CWMNs have experimented with a variety of models for sustainability and governance in order to resolve long-term operational and maintenance issues, especially when many lay practitioners are involved. The majority relies on voluntary work from activists and on contributions from their members to offer Internet connectivity; usually, node owners buy their hardware: radio, antenna, cables (e.g. Freifunk, Ninux.org, and S-Net). Guifi.net has been asking members (including commercial operators) to adopt a specific network licence, subscribe to an arbitrator agreement and contribute with a fee which includes network upgrading and development and also maintenance. This is carried by senior members, professionals who also offer other telecommunication services over the network (landline telephone or IT support) [4]. Red Hook mesh in NYC, instead, offers a model where installation and maintenance are provided by Digital Stewards: these are young residents from the local public housing employed in a paid fellowship (20 hours a week at \$8.75 an hour) [5]. Whatever the model adopted, every group involved in CWMN maintains a local weekly or monthly assembly for face-to-face discussion and problem-solving.

\section{WMNs of Things}

While traditionally the Internet has facilitated interaction between humans, the current landscape starts being dominated by the Internet of Things (IoT). These 'things' typically have a communication interface, processing and storage units, sensors for detection of environmental changes or services to other clients. The combination of short-range wireless signals and the wider cellular network can provide wireless connectivity for local devices to exchange data to the Internet. Applications using WMNs cannot rely on the lower routing protocol security and need to implement trust negotiation, authentication and credential 
mechanisms to grant the integrity of communications [6]. As the mesh extends in space and by number of users (horizontal and vertical scalability), there is a risk of increased latency (slow response). Network latency can raise serious issues such as interference problems, data communication errors, and others related to architecture, resource allocation, mobility management, quality of service provisioning, and security [7].

To this regard, the high-tech industry is piloting new solutions such as 5G connectivity and the so-called World Wide Herd (WWH), ${ }^{1}$ which aims to analyse large decentralised datasets on site, without requiring the data to be moved to a single location. Thus, IoT mesh networks are increasingly vital to the data extractive practices of Big Tech (Dell estimates 200 billion connected devices in 2031), but they can be exposed to many security vulnerabilities due to their multi-hop nature, lack of physical protection, dynamic topology, and ad-hoc connectivity.

See Also: wbeos0563, wbeose011.pub2, wbeos0942, wbeos0997, wbeosn014.pub2, wbeost011.

\section{References}

A. Medosch, Freie Netze. Hannover: Heise Heinz, 2003. Polygon, SNET: Cuba's Underground Internet \& The Rise of Competitive Gaming. . M. Portmann and A. A. Pirzada, "Wireless mesh networks for public safety and crisis management applications,” IEEE Internet Comput., vol. 12, no. 1, 2008.

R. Baig, R. Roca, L. Navarro, and F. Freitag, "guifi.net: a network infrastructure commons," in Proceedings of the Seventh International Conference on Information and Communication Technologies and Development - ICTD '15, Singapore, Singapore, 2015, pp. 1-4.

N. Cohen, “Red Hook’s Cutting-Edge Wireless Network,” The New York Times, 20-Dec2017.

A. Sgora, D. D. Vergados, and P. Chatzimisios, "A survey on security and privacy issues in Wireless Mesh Networks,” Secur. Commun. Netw., vol. 9, no. 13, pp. 1877-1889, 2016. I. Yaqoob, I. A. T. Hashem, Y. Mehmood, A. Gani, S. Mokhtar, and S. Guizani, "Enabling Communication Technologies for Smart Cities," IEEE Commun. Mag., vol. 55, no. 1, pp. 112-120, Jan. 2017.

1https://dellemcstudy.blogspot.com/2018/07/distributed-analytics-meets-distributed.html 
